EPJ Web of Conferences 61, 01004 (2013)

DOI: 10.1051/epjconf/ 20136101004

(C) Owned by the authors, published by EDP Sciences, 2013

\title{
The M87 Jet
}

\section{"Rosetta Stone" of AGN Jets}

\author{
Masanori Nakamura ${ }^{1, a}$ and Keiichi Asada ${ }^{1}$ \\ ${ }^{1}$ Institute of Astronomy and Astrophysics, Academia Sinica, 11F of Astronomy-Mathematics Building, AS/NTU No 1, Sec 4, \\ Roosevelt Rd., Taipei 10617, Taiwan
}

\begin{abstract}
We investigate the structure and dynamics of the M87 jet based on multi-frequency VLBI observations and MHD jet theories. Millimeter VLBI cores are considered as innermost jet emissions. The jet structure up to $\sim 10^{5} r_{\mathrm{s}}$ is described as a parabolic streamline, indicating the lateral expansion under a confinement by the stratified ISM. Thus, the jet collimation maintains in five orders of magnitude in the distance starting from the vicinity of the supermassive black hole $(\mathrm{SMBH})$, less than $10 r_{\mathrm{s}}$. We here examine the jet parabolic structure in order to identify the property of a bulk acceleration; observed sub-to-superluminal motions indicate an MHD acceleration from non-relativistic to relativistic regimes. We propose that the M87 jet consists of Poynting-flux dominated flows, powered by nonlinear torsional Alfvén waves. Future sub-mm VLBI observations play an important role in resolving the origin of the M87 jets.
\end{abstract}

\section{Introduction}

How do the acceleration and collimation, as well as the formation process, take place in astrophysical jets? Although electromagnetic and/or magnetohydrodynamic (MHD) mechanisms are frequently invoked to extract energy and momentum from the compact object and/or the accretion disk [e.g., 1-5], none of the real astrophysical jet systems have yet been examined to confirm these theoretical properties in a quantitative manner.

M 87 is one of the nearest active galaxies [16.7 Mpc; 6] that exhibit relativistic outflows. The mass of the central supermassive black hole $(\mathrm{SMBH}) M_{0}$ is measured with a range $3.2 \times 10^{9} M_{\odot}$ [7] to $6.6 \times 10^{9} M_{\odot}$ [8]. This largest mass gives an apparent angular size $\sim 8 \mu$ as for the Schwarzschild radius $r_{\mathrm{s}} \equiv 2 G M_{\bullet} / c^{2}$. This galaxy therefore provides a unique opportunity to study the relativistic outflow with the highest angular resolution by the very long baseline interferometry (VLBI) technique in units of $r_{\mathrm{s}}$. Extended synchrotron emissions of one-sided jet, emerging from the nucleus, have been well-studied at multiple wavelengths from radio to X-ray bands [9-21], which cover a spatial scale $\sim 0.4$ mas -14 arcsec. With viewing angle $14^{\circ}$ [22], it corresponds to $\sim 2 \times 10^{2} r_{\mathrm{s}}-7 \times 10^{6} r_{\mathrm{s}}$ in de-projection.

In the standard picture of VLBI jets, the compact radio core (VLBI core) is widely believed as the throat of a diverging (with a constant opening angle), conical jet [23]. Alternatively, [24] proposed that a VLBI core is identified as the first re-collimation Mach disk-oblique shock system. In general, the VLBI core of a Blazar jet at mm wavelength is located at $10^{4-6} r_{\mathrm{s}}$ where the synchrotron emis-

\footnotetext{
a e-mail: nakamura@asiaa.sinica.edu.tw
}

sion is completely optically thin (where the optical depth $\tau(v)$ is fallen to $\sim 1$ ), which corresponds to the turnover in the spectrum [25]. The jet acceleration and collimation zone is speculated in the upstream of the mm-wave radio core [26].

Very recently, there have been several attempts to examine the inner jet structures on M87: i) Hada et al. (2011) [27] performed the core shift measurement by using multifrequency, phase-referencing Very Long Baseline Array (VLBA) observations, indicating that the $43 \mathrm{GHz}$ VLBI core is located at $\sim 20 r_{\mathrm{s}}$ from the central engine (presumably, the SMBH and/or accretion disk). They conclude that the measured frequency dependence of the core shift $\propto v^{-0.94 \pm 0.09}$ is in good agreement with a synchrotron selfabsorbed jet $[23,28]$. ii) Asada \& Nakamura (2012) [29, hereafter AN12] investigated the structure of the M87 jet, from milliarcsec (mas) to arcsec scales by utilizing the images taken with EVN, MERLIN, and VLBA. AN12 reveal that the jet maintains a "parabolic" shape $z \propto r^{1.73 \pm 0.05}$ in a de-projected distance of $10^{2}-10^{5} r_{\mathrm{s}}$, where $r$ is the radius of the jet emission and $z$ is the axial distance from the core. iii) Doeleman et al. (2012) [30] conducted the Event Horizon Telescope (EHT) observation at a wavelength of $1.3 \mathrm{~mm}$, deriving the size of the $230 \mathrm{GHz}$ VLBI core to be $5.5 \pm 0.4 r_{\mathrm{s}}$. This is smaller than the diameter for the innermost stable circular orbit (ISCO) of a retrograde accretion disk, suggesting that the M87 jet may be powered by a prograde accretion disk around a spinning SMBH.

In the present paper, we argue the innermost region of the M87 jet by using VLBA archival data at $43 / 86 \mathrm{GHz}$. We apply the standard picture of the VLBI core (as an innermost jet at giving frequency) to M87, one of the most resolved AGN jets, to inspect the feasibility of the physical 
interpretation of the core. We also discuss the jet acceleration and collimation in M87. Throughout the paper, we use the de-projected distance along the jet from the central $\mathrm{SMBH}$ in units of $r_{\mathrm{s}}$. Note that 1 mas corresponds to 517 $r_{\mathrm{s}}$ in de-projection.

\section{Global Structure: A Parabolic Stream}

\subsection{Archival Data and Data Reduction}

We analyzed archival data (BW088 and BJ045) of VLBA observations at 43 and $86 \mathrm{GHz}$. VLBA observation at 43 $\mathrm{GHz}$ was carried out on 2007 January 27 at 43.127 and 43.135 GHz using all 10 stations of the VLBA except the Kitt Peak station. VLBA observation at $86 \mathrm{GHz}$ was carried out on 2007 January 17 at 86.253 and $86.269 \mathrm{GHz}$ using all stations of the VLBA except the Saint Croix and Hancock stations. Each IF has $16 \mathrm{MHz}$ bandwidth in both observations. The data were correlated at the VLBA correlator at Socorro.

An a priori amplitude calibration for each station was derived from the system temperatures measured during observations and antenna gain curve information from standard files. Opacity correction was made during the amplitude calibration process using the AIPS task APCAL. Fringe fitting was performed on each IF and polarization independently using the AIPS task FRING. After delay and rate solutions were determined, the data were averaged over $12 \mathrm{~s}$ in each IF and self-calibrated using Difmap. We note that the VLBA observations were conducted with dual polarization, and we independently reduced the data at $86 \mathrm{GHz}$ at both polarizations and confirmed consistency of the obtained images.

\subsection{Results}

We define the core with a Gaussian model that was fitted to the innermost bright region in all the images. Measured sizes of the VLBI core at $43\left(\right.$ core $\left._{43}\right)$ and $86 \mathrm{GHz}$ (core 86 ) were $182 \pm 53 \mu$ as and $100 \pm 55 \mu$ as, corresponding to $23 \pm 7 r_{\mathrm{s}}$ and $13 \pm 7 r_{\mathrm{s}}$, respectively. The size of core 86 is in good agreement with $99 \pm 21 \mu$ as in the previous GMVA observation [31]. Also we remark that the size of the VLBI core at $230 \mathrm{GHz}\left(\right.$ core $\left._{230}\right)$ is derived by the EHT observation as $5.5 \pm 0.4 r_{\mathrm{s}}$ in the correlated flux density analysis by circular Gaussian models [30].

Figure 1 shows the radius of the jet as a function of the de-projected distance from the central SMBH. Current innermost emissions of the M87 jet lie at $\sim$ few $\times 10^{2} r_{\mathrm{s}}$ from the central SMBH, which have been observed by $43 \mathrm{GHz}$ VLBA observations. However, if we consider a hypothesis, which has been introduced by [23], that the VLBI core is the most upstream of the optical thin synchrotron emission at a given frequency [thus a position of the "selfabsorbed core" depends on an observed frequency: e.g., 28], we can examine the further innermost jet structure by using VLBI cores with $43 \mathrm{GHz}$ and higher frequencies.

In order to determine an offset position in the jet axial direction (from the core position to the central engine), we follow the formula in [27]. An absolute offset
$\Delta z$ can be determined as a function of the frequency as $\Delta z(v / \mathrm{GHz})=A v^{-\alpha}$ (mas), where $\alpha=0.94 \pm 0.09$ and $A=1.40 \pm 0.16$. We therefore estimate $\Delta z(43 \mathrm{GHz})=$ $21.2 \pm 6.2 r_{\mathrm{s}}, \Delta z(86 \mathrm{GHz})=10.9 \pm 4.7 r_{\mathrm{s}}$, and $\Delta z(230$ $\mathrm{GHz})=4.34 \pm 2.17 r_{\mathrm{s}}$, respectively. As is shown in Fig. 1 , three points of VLBI cores (core 43 , core 86 , and core 230 ) are plotted under our assumption that the axial offset position at infinite frequency $\Delta_{\infty}(\rightarrow 0)$ is the location where the SMBH and/or accretion disk plane exist. Note that VLBA core sizes at 5.0 and $8.4 \mathrm{GHz}$ coincide with the jet width derived in VLBA observations at $43 \mathrm{GHz}$ [32]; thus, it may be reasonable to interpret the frequency-dependent VLBI core as an innermost synchrotron emission where the jet emissions become optically thin.

The parabolic jet seems to follow a single power-law streamline with nearly five orders of magnitude in distance. One, however, has to bear in mind the following questions: where does jet origin exist and how does its non-thermal emission is initiated at some higher frequency? It is nevertheless useful for us to examine the nature of the jet parabolic structure-how it is maintained under the stratified ISM in the dominant gravitational potential by the central SMBH. Expected positions of sub$\mathrm{mm}$ VLBI cores at $340 \mathrm{GHz}$ and $690 \mathrm{GHz}$ are also plotted on Fig. 1 for our references. Thus, we suggest future sub$\mathrm{mm}$ VLBI experiments play an important role in studying the jet footpoint.

\section{Dynamics: MHD Acceleration?}

\subsection{Proper Motions As A trace of Bulk Flows}

In a general framework on asymptotic evolutions of MHD jets in parabolic streamlines, both bulk acceleration and collimation are expected to take place simultaneously. In this section, we investigate the jet acceleration; how it is correlated to the parabolic structure derived in $\$ 2$. one of the useful tools to study the jet kinematics is the direct measurement of a proper motion (an "apparent" speed $V_{\text {app }}$ of moving components) by the multi-epoch observations. Let $\theta_{\mathrm{v}}$ be a viewing angle between our line of sight and an "intrinsic" speed $V_{\text {int }}$ along the jet. Then $V_{\text {app }}$ is corrected in the units of the speed of light $c(\beta=V / c)$ through the usual relationship: $\beta_{\text {app }}=\beta_{\text {int }} \sin \theta_{\mathrm{v}} /\left(1-\beta_{\text {int }} \cos \theta_{\mathrm{v}}\right)$.

Proper motions of the M87 jet in the parabolic region ( $\lesssim 10^{5} r_{\mathrm{s}}$ ) have been probed by VLBI and HST observations during the past two decades [e.g., 9, 13, 21, 40] as shown in Fig. 2, exhibiting a series of subluminal motions. Note that a peak of apparent motions, that is, superluminal $\beta_{\text {app }} \sim 6$, is located at around the HST- 1 complex [13], where the jet has a structural transition from the parabolic to conical streamline as shown in AN12. As is clearly seen in Fig. 2, it is remarkable that observed apparent speed keeps increasing by almost two orders of magnitude at $z=10^{2}-10^{5} r_{\mathrm{s}}$, where the jet structure is parabolic.

In the literature as listed above, these subluminal motions upstream of HST-1 have been generally interpreted as stationary patterns as standing shocks and/or some modes of plasma instabilities. This may be because one could consider the M87 jet structure as a cylindrical or 


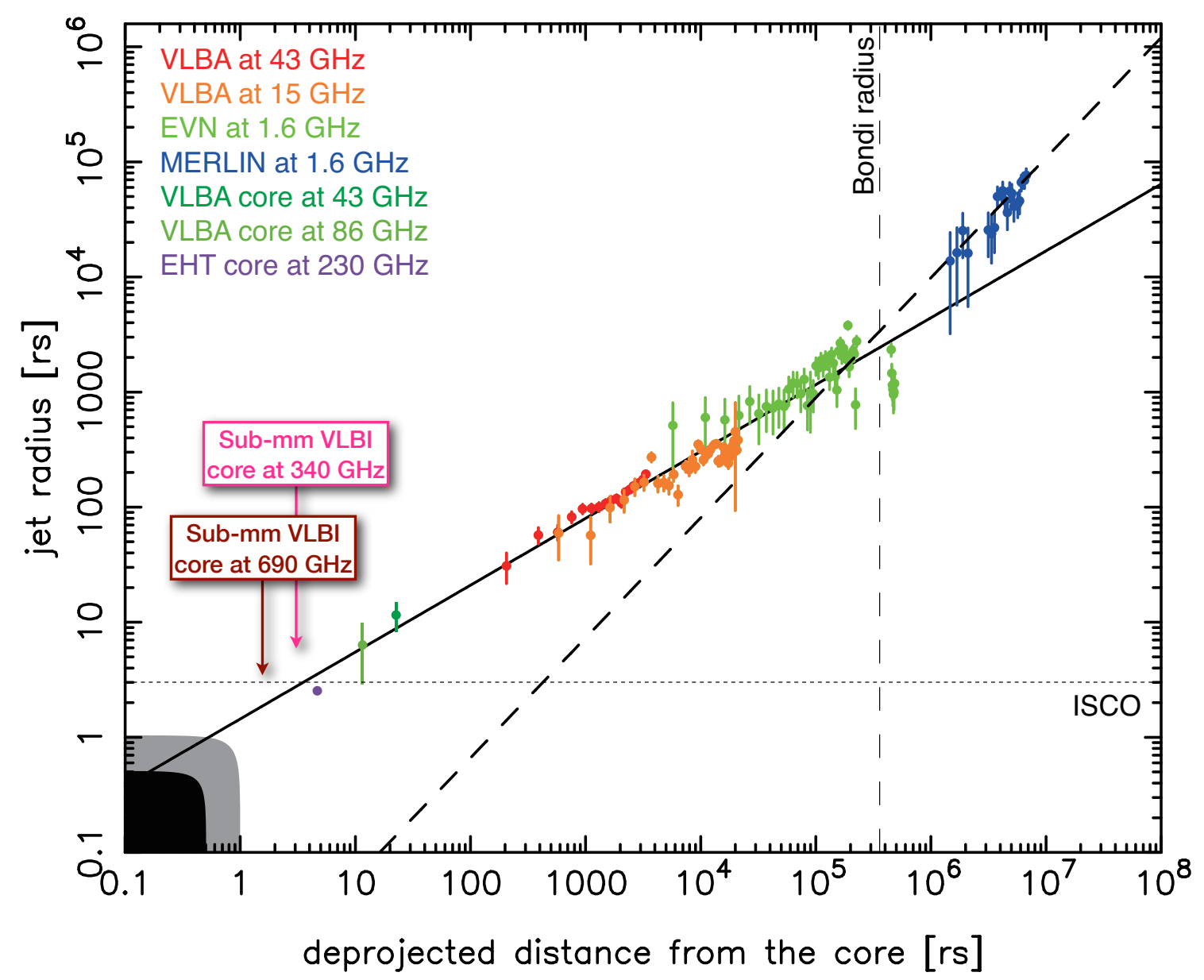

Figure 1. Distribution of the radius $(r)$ of the jet as a function of the de-projected distance $(z)$ from the core in units of $r_{\mathrm{s}}: z \propto r^{a}$. Three data points of VLBI cores (at 43, 86, and $230 \mathrm{GHz}$ ) are added as the most inner jet emissions at each frequency. The solid line is the linear least-square for data points except three inner cores (VLBA at $43 / 86 \mathrm{GHz}$ and EHT at $230 \mathrm{GHz}$ ), indicating the parabolic streamline with a power-law index $a$ of $1.73 \pm 0.05$. On the other hand, the dashed line indicates the conical streamline with $a$ of $0.96 \pm 0.1$. HST-1 is located around $5 \times 10^{5} r_{\mathrm{s}}$. Readers can refer to Fig. 2 in [29] for detailed description. Expected positions of sub-mm VLBI cores at $340 \mathrm{GHz}$ and $690 \mathrm{GHz}$ are also plotted.

conical structure [e.g., 21]. When the supersonic, hydrodynamic jet, which is highly over-pressured against the ambient ISM, it forms a conical shape and is causally disconnected from its driving engine [33]. Under a shallow decrease of the ambient ISM, a re-collimation shock will be formed due to the pressure imbalance between the inside and outside of the jet [34]. Or perhaps, growing plasma instability and its related turbulence may be speculated. However, we suggest neither would be the case in M87; the jet structure is parabolic so that the jet outer edge still has a causal contact with its interior, where the jet could be magnetically accelerated up to over $10^{5} r_{\mathrm{s}}$ scale.

Lister et al. (2009) [35] investigate the parse-scale AGN jet kinematics in the complete MOJAVE survey; a median rms dispersion of apparent speeds in the overall sample is three times larger than that value within an individual jet. Two-sided jets in relatively low-luminosity and nearby radio galaxies provide notable evidence that apparent motions in the jet and counter jet are correlated. This survey may give an additional insight that there is a characteristic flow that describes each jet; apparent motions are not simply (shock/instability) patterns, which propagate slowly and/or relatively stable, but do indeed represent underlying bulk flows. Even $V_{\text {app }}$ is corrected by a viewing angle, random distribution of intrinsic speeds at two sides should be generally expected no matter whether the jet and counter jet are intrinsically symmetric (similar kinematics of each side due to a common origin of the jet initiation). In at least NGC 1052 and Cygnus A, Lister et al. (2009) [35] confirm a one-to-one association between features on the two sides, supporting a hypothesis that observed proper motions reflect the real jet flow. It would seem far more natural to have $V_{\text {int }}$ tied to the jet (fluid) bulk speed $V$ as $\beta_{\text {int }} \lesssim \beta[11]$.

Another important remark is that MHD jets, which are powered by torsional Alfvén waves [TAWs 36, 37], are intrinsically robust compared to purely hydrodynamic jets regarding nonlinear wave perturbations. When wave amplitudes are no longer small, most disturbances such as a (magneto-)sonic wave cease to propagate as a wave with a constant profile, but instead distort in time (steepening into a shock). However, Alfvén waves can continue to 


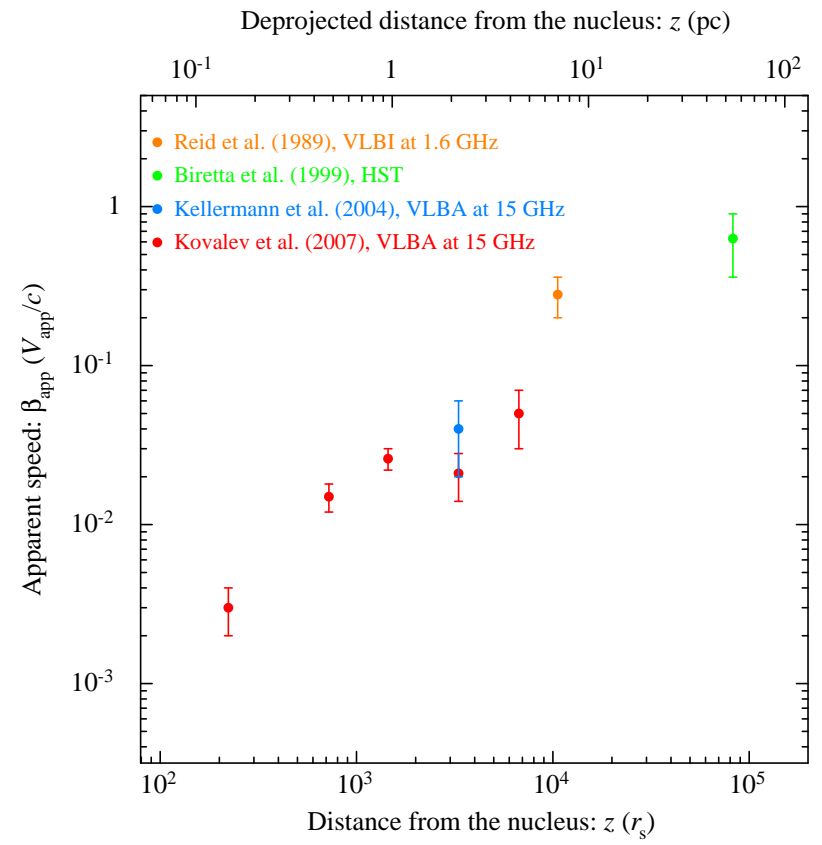

Figure 2. Distribution of the apparent speed $\beta_{\text {app }}\left(=V_{\text {app }} / c\right)$ as a function of distance from the nucleus in the unit of $r_{\mathrm{s}}$ (note the upper scale denotes the de-projected distance in parsecs).. Measurements by VLBA at $15 \mathrm{GHz}$ [red and blue; 21, 40, respectively], global VLBI at 1.6 GHz [orange; 9], and HST [green; 13].

propagate without distortions even when their amplitudes are large [38]. Recently, this unusual, but very interesting property is also verified in the relativistic Alfvénic perturbations [39]. We thus suggest that observed subluminal patterns in M87 may correspond to slowly, but presumably non-relativistic fluids that are magnetically accelerated along the jet parabolic streamline.

\subsection{Bulk Acceleration of the Trans-Alfvénic Flow}

In order to inspect the property of the jet acceleration in the trans-Alfvénic regime, let us follow the wave dynamics of TAWs propagating along a global poloidal magnetic flux tube: $B_{p} \Sigma=\Psi$ (const.), where $\Sigma\left(\propto r^{2}\right)$ is a cross section of the flux tube and $\Psi$ is the magnetic flux [37].

Under the WKB approximation, the conservation of the energy flux density of the TAW is considered through a circular (cross-sectional) area around the rotation axis in cylindrical coordinates $(r, \phi, z)$ as $\rho \delta V_{\phi}^{2} V_{\mathrm{A}} / B_{p}=$ const., where $\rho, \delta V_{\phi}$, and $V_{\mathrm{A}} \equiv B_{p} /(4 \pi \rho)^{1 / 2}$ are the gas density, azimuthal component of the velocity field (a transverse displacement against the poloidal magnetic line of force drives a TAW), and Alfvén speed, respectively. Perturbed quantities in the transverse direction satisfy $\delta V_{\phi}=-\delta B_{\phi} / \sqrt{4 \pi \rho}$, which is another property of the shear Alfvén wave [38]. Thus, TAWs can be characterized as an equality of ratios between poloidal and toroidal components in the magnetic field, $\delta B_{\phi} / B_{p}=-\delta V_{\phi} / V_{\mathrm{A}}$. Note that the wave amplitudes $\left(\delta B_{\phi}\right.$ and $\left.\delta V_{\phi}\right)$ are not necessarily small, but can be arbitrarily finite.
Considering the trans-Alfvénic, non-relativistic MHD (NRMHD) bulk flow powered by nonlinear TAWs, orderof-magnitude estimations of the one-dimensional ( $z$ ) equation of motion give $V_{z} \sim B_{\phi}^{2} /\left(4 \pi \rho V_{\mathrm{A}}\right)$. By combining above equations, we can derive $V_{z} \sim B_{p}^{-1}$. Thus, the terminal velocity of the TAW-driven MHD jet model with an initially uniform axial field ( $B_{p}=$ const .) is a constant with an order of trans-Alfvénic speed. On the other hand, the terminal velocity of a TAW-driven MHD jet with a nonuniform axial field $\left(B_{p} \neq\right.$ const.) can be super-Alfvénic (even super-fast magnetosonic) in the downstream, suggesting $V_{z} \propto z^{2 / a}$. Again, $a$ denotes the power-law index of the parabolic streamline $z \propto r^{a}(1<a \leq 2)$ of a magnetic flux tube. In the specific case of a purely parabolic stream with $a=2$, we note the trans-Alfvénic jet may exhibit that the flow speed increases linearly as a function of the distance $V_{z} \propto z$, which can be observed in NRMHD simulations [e.g., 41, 42].

In Fig. 3, we show the bulk speed $\beta(=V / c)$ corrected by $\beta_{\text {app }}$ with $\theta_{\mathrm{v}}=14^{\circ}$ as a function of the de-projected distance, showing a feature of the acceleration from $\beta \simeq 10^{-2}$ to 0.7 in the range of $z=10^{2}-10^{5} r_{\mathrm{s}}$. A distribution of $\beta$ exhibits a linear increase as a function of $z$ as $\beta \propto z$ around $z \simeq 10^{2}-10^{3} r_{\mathrm{s}}$, suggesting a trans-Alfvénic flow. In order to fit the data points and derive a solid picture, more sampling from this region is desired. Therefore, higher angular resolution and higher sensitivity observation with detailed analysis to identify more components and trace the proper motions are important. Beyond $z \gtrsim 10^{3} r_{\mathrm{s}}$, it seems that the bulk acceleration is weakened slightly, indicating that the jet may transit from trans-Alfvénic to trans-fast magnetosonic, but the underlying flow is still accelerated toward a relativistic regime $\beta \lesssim 1$.

\section{Discussions}

\subsection{Nature of the Upstream Flow within $100 r_{\mathrm{s}}$}

We first discuss the hot accretion disk as an origin of the MHD outflow in M87. In the ADAF (RIAF) model $\left(C_{s} \sim\right.$ $V_{\mathrm{K}}$, where the Keplerian speed $V_{\mathrm{K}}$ is equal to $\left.c / \sqrt{2 r / r_{\mathrm{s}}}\right)$, the disk (ion) temperature is considered as nearly virial, $T_{\mathrm{i}}=5.4 \times 10^{12}\left(r / r_{\mathrm{s}}\right)^{-1} \mathrm{~K}$ [43]. Given $r \sim 10 r_{\mathrm{s}}$ in Fig. 1 (where VLBI cores are located), the sound speed $C_{s} \simeq 0.28 c$ is also comparable to the local escape speed $V_{\text {esc }}=c / \sqrt{r / r_{\mathrm{s}}} \sim 0.3 c$. Therefore, we would seek much faster motions in the downstream than measured speeds $0.01 c-0.1 c$ at $10^{2}-10^{3} r_{\mathrm{s}}$ based on more than $10 \mathrm{yr}$ of VLBA observations at $15 \mathrm{GHz}$ [21, see also Fig. 2], which are considered as the bulk speed in $\S 3.1$.

If the M87 jet indeed originated from the surface of RIAFs $\left[B_{\phi} \sim B_{p}\right.$; e.g., 5], we may not expect a standard magneto-centrifugal acceleration process [2], but favor an initially trans-Alfvénic flow [see also 41]. Thus, this may give some constraint on the required MHD jet model with an initial jet speed with an order of $\sim 0.1 c^{1}$ at the jet footpoint.

${ }^{1}$ Ly et al. (2007) [20] reported possible but transient features $V_{\text {app }}=$ $(0.25-0.40) c$ located at about 3 mas based on just two of five total epochs ( $\sim 8$ months apart) of VLBA and global VLBI observations at $43 \mathrm{GHz}$; 


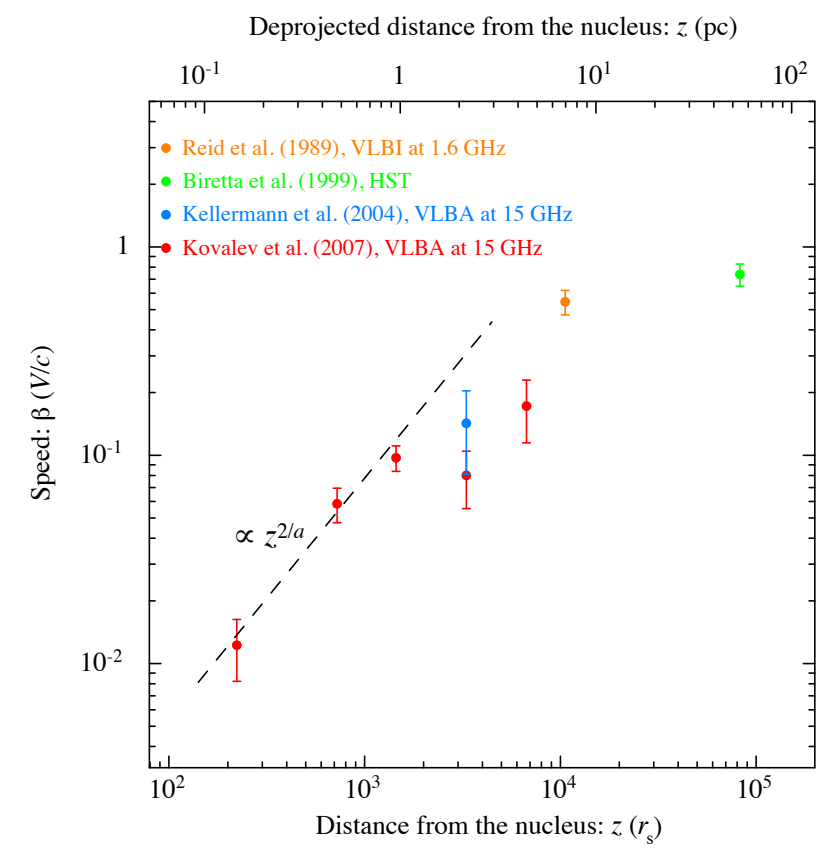

Figure 3. Distribution of the bulk speed $\beta(=V / c)$ as a function of distance from the nucleus in the unit of $r_{\mathrm{s}}$ (note the upper scale denotes the de-projected distance in parsecs). Measurements are illustrated in the same manner as Fig. 2. The dashed line represents $\beta \propto z^{2 / a}$ with $a=1.73$; note it is not a fit to data points, but is derived analytically in $\$ 3.2$.

Next, we discuss the Kerr black hole as an origin of the MHD outflow in M87. The outermost sheath of the ergosphere-driven jet exhibits non-relativistic motion $\gamma \simeq 1$ at $\sim 5 \times 10^{3} r_{\mathrm{g}}$ as seen in GRMHD simulations [44]; from a polar angle cross-sectional distribution of the gas density, we can find that the outermost sheath has much higher mass loading (about four orders of magnitude) than the innermost jet spine. This may cause an inefficient bulk acceleration at least up to this spatial scale. A matter piling up at the outer surface of the jet may also be responsible for observed edge-brightened features in M87. The location of the fast magnetosonic surface is about a few of hundred $r_{\mathrm{s}}$ near the surface layer of the ergosphere-driven jet where the jet is still Poynting-flux dominated.

Thus, we may speculate a behavior of the jet at sub- to trans-Alfvénic region $z \lesssim 100 r_{\mathrm{s}}$ as a magneto-centrifugal driving process (by not a disk rotation, but an ergosphere) similar to and then the jet seems to be powered by TAWs (the toroidal magnetic pressure gradient force) at transAlfvénic regions. Note that a magneto-centrifugal driving allows the flow to start at a very slow speed $V_{0}$ compared to the rotational motion of the magnetic field [41]. In the case of non-relativistic Keplerian disk, $V_{\mathrm{K}} / V_{0} \simeq 300$ can be obtained in steady, axisymmetric NRMHD outflow solutions $[45,46]$. Thus, even for relativistic field rotations with $0.1 c-c$ near the black hole, we may speculate an initial jet speed with $V_{0} \ll 0.1 c$ at the jet footpoint.

these components would not be present in other epochs. It may be still unclear whether a detection of an order of $V=0.5 c-0.6 c$ (at $\simeq 1.5 \times$ $10^{3} r_{\mathrm{s}}$ in de-projection) will support an RIAF-originated jet scenario.
One of the crucial tests to constrain the nature of the upstream flow within $100 r_{\mathrm{s}}$ is to image extended jet emissions from the VLBI core. We are conducting new GMVA observations at $86 \mathrm{GHz}$ in order to resolve the innermost jet structure at a few $10^{1-2} r_{\mathrm{s}}$ scales.

\subsection{An Offset of the Jet Footpoint from the Black Hole and Effect on Core Shifts}

In Fig. 1, we show that three additional data points of VLBI cores seem to fairly follow a parabolic streamline derived by AN12, implying that the jet maintains a single non-conical structure with nearly five orders of magnitude in distance. We here discuss about limitations of a frequency depending VLBI core shift measurement to seek the true origin of the M87 jet. The median values of the VLBI core shifts in AGN jets are reported as 0.080 mas [47] and 0.128 [48] between $15.4-8.1 \mathrm{GHz}$. A scale of $\sim 0.1$ mas corresponds to $1-10 \mathrm{pc}$ in de-projection for typical blazars with a small viewing angle $\theta_{\mathrm{v}} \sim 5^{\circ}$, which is equivalent to $10^{3-4} r_{\mathrm{s}}$ with $M_{\bullet}=10^{9} M_{\odot}$. Therefore, if one applies this method to blazars at even higher frequencies, an axial offset between the position of the SMBH (the origin) and/or accretion disk (the equatorial plane) and the jet foot point may be ignorable.

On the other hand, in the case of M87, the value of the VLBI core shift between 15.4 and $8.4 \mathrm{GHz}$ is similar [ $\sim 0.1$ mas; 27], but it corresponds to $\sim 10 r_{\mathrm{s}}$, approaching a limit to apply the method of frequency-dependent VLBI core shift measurements. By using the formula in Hada et al. (2011) [27], we derive $\Delta z(230 \mathrm{GHz})=4.3 \pm 2.2 r_{\mathrm{s}}$ (see $\S 2.2$ ), which may be comparable to the so-called stagnation surface (SS) seen in GRMHD simulations [e.g. 44]. If we consider the MHD jet launching from the rotating SMBH magnetosphere [1], the SS (where the poloidal velocity component changes the sign $-/+$ : the inflow onto the SMBH inside the SS, while the outflow as a jet outside the SS) has an offset from the SMBH. The location of the SS depends on a spin of the black hole: the balance between the gravity and the centrifugal forces along a poloidal magnetic field in the co-rotating frame with the line of force.

McKinney (2006) [44] prescribes a black hole spin of $j=0.9375$ and the SS is located at around 2-3r $r_{\mathrm{s}}$. A lower spin will put the radius of the SS further downstream $>\Delta z$ $(230 \mathrm{GHz})$. Even if we consider that the MHD jet originates from an RIAF-type accretion disk $H \sim r, r \gtrsim 2.5 r_{\mathrm{s}}$ ( $H$ is the disk scale height) [the lensed ISCO radius for the prograde spinning $j=1 ; 30$ ], then the disk surface is presumably the SS so that the situation will be unchanged. Thus, it may be challenging to explore the jet footpoint in M87 by using VLBI cores at higher frequencies. If we take an offset of the SS into consideration, three VLBI cores may not be located on a parabolic streamline in Fig. 1. Thus, future sub-mm VLBI imaging of the jet footpoint together with the SMBH shadow is crucial. 


\section{Summary}

Following Asada \& Nakamura (2012) [29], we further investigate the parabolic jet structure in M87 with additional data points of VLBI cores and examine theoretical aspects of MHD jets. We here propose a hypothesis that the observed subluminal motions on $0.1-100 \mathrm{pc}$ scales in projection (corresponding to $\sim 10^{2}-10^{5} r_{\mathrm{s}}$ ) show the MHD bulk acceleration in a non-relativistic regime although they have been interpreted during past decades as standing shocks and/or some patterns of plasma instabilities in supersonic jets. Based on our analysis, it is reasonably described as a consequence of Poynting fluxdominated flows, powered by large-amplitude, nonlinear TAWs in a parabolic streamline.

An origin of the M87 jet is also discussed based on our current understanding in observations and theories although we will need further observational constraints of the nature of the upstream flow within $100 r_{\mathrm{s}}$. We furthermore suggest that it may be challenging to seek the true jet origin only by using VLBI core shift measurements in M87. Thus, we encourage future observations with a high sensitivity such as a space VLBI and/or mm/sub-mm VLBI at $86 \mathrm{GHz}$ or higher.

\section{References}

[1] Blandford, R. D., \& Znajek, R. L., MNRAS, 179, 433 (1977)

[2] Blandford, R. D., \& Payne, D. G., MNRAS, 199, 883 (1982)

[3] Pelletier, G., \& Pudritz, R. E., ApJ, 394, 117 (1992)

[4] Meier, D. L., ApJ, 522, 753 (1999)

[5] Meier, D. L., ApJ, 548, L9 (2001)

[6] Jordán, A., et al., ApJ, 634, 1002 (2005)

[7] Macchetto, F., et al., ApJ, 489, 579 (1997)

[8] Gebhardt, K., et al., ApJ, 729, 119 (2011)

[9] Reid, M. J., et al., ApJ, 336, 112 (1989)

[10] Owen, F. N., et al., ApJ, 340, 698 (1989)

[11] Biretta, J. A., et al., ApJ, 447, 582 (1995)

[12] Perlman, E. S., et al., AJ, 117, 2185 (1999)
[13] Biretta, J. A., et al., ApJ, 520, 621 (1999)

[14] Junor, W., et al., Nature, 401, 891 (1999)

[15] Marshall, H. L., et al., ApJ, 564, 683 (2002)

[16] Wilson, A. S., \& Yang, Y., ApJ, 568, 133 (2002)

[17] Perlman, E. S., \& Wilson, A. S., ApJ, 627, 140 (2005)

[18] Harris, D. E., et al., ApJ, 640, 211 (2006)

[19] Cheung, C. C., et al., ApJ, 663, L65 (2007)

[20] Ly, C., Walker, et al., ApJ, 660, 200 (2007)

[21] Kovalev, Y. Y., et al., ApJ, 668, L27 (2007)

[22] Wang, C.-C., \& Zhou, H.-Y., MNRAS, 395, 301 (2009)

[23] Blandford, R. D., \& Königl, A., ApJ, 232, 34 (1979)

[24] Daly, R. A., \& Marscher, A. P., ApJ, 334, 539 (1988)

[25] Jorstad, S. G., et al., AJ, 134, 799 (2007)

[26] Marscher, A. P., et al., Nature, 452, 966 (2008)

[27] Hada, K., et al., Nature, 477, 185 (2011)

[28] Königl, A. 1981, ApJ, 243, 700 (1981)

[29] Asada, K., \& Nakamura, M., ApJ, 745, L28 (2012)

[30] Doeleman, S. S., et al., Science, 338, 355 (2012)

[31] Lee, S.-S., et al., AJ, 136, 159 (2008)

[32] Hada, K., these proceedings, (2013)

[33] Zakamska, N. L., ApJ, 679, 990 (2008)

[34] Sanders, R. H., ApJ, 266, 73 (1983)

[35] Lister, M., et al., AJ, 138, 1874 (2009)

[36] Uchida, Y., \& Shibata, K., PASJ, 37, 515 (1985)

[37] Shibata, K., \& Uchida, Y., PASJ, 37, 31 (1985)

[38] Priest, E. R., Solar Magneto-hydrodynamics, (Dordrecht, Reidel, 1981), 160-161

[39] Heybaerts, J., et al., A\&A, 542, A128 (2012)

[40] Kellermann, K. I., et al., 2004, 609, 539 (2004)

[41] Contopoulos, J., ApJ, 450, 617 (1995)

[42] Ouyed, R., \& Pudritz, R. E., ApJ, 482, 712 (1997)

[43] Narayan, R., \& Yi, I., ApJ, 428, 13L (1994)

[44] McKinney, J. C., MNRAS, 368, 1561 (2006)

[45] Kudoh, T., \& Shibata, K., ApJ, 474, 362 (1997)

[46] Vlahakis, N., et al., MNRAS, 318, 417 (2000)

[47] Sokolovsky, K. V., et al., A\&A, 532, A38 (2011)

[48] Pushkarev, A. B., et al., A\&A, 545, A113 (2012) 